\title{
A Cholecystokinin-releasing Factor Mediates Ethanol-induced Stimulation of Rat Pancreatic Secretion
}

\author{
A.K. Saluja, L. Lu, Y. Yamaguchi, B. Hofbauer, M. Rünzi, R. Dawra, M. Bhatia, and M.L. Steer \\ Department of Surgery, Beth Israel Hospital, Harvard Medical School and Harvard Digestive Diseases Center, Boston, \\ Massachusetts 02215
}

\begin{abstract}
The mechanisms by which short-term ethanol administration alters pancreatic exocrine function are unknown. We have evaluated the effects of ethanol administration on pancreatic secretion of digestive enzymes. In our studies, anesthetized as well as conscious rats were given ethanol at a rate sufficient to cause the blood ethanol concentration to reach levels associated with clinical intoxication. Ethanol was administered over a 2 -h period during which blood ethanol levels remained stably elevated. We report that intravenous administration of ethanol results in a transient increase in pancreatic amylase output and plasma cholecystokinin (CCK) levels. The ethanol-induced increase in amylase output can be completely inhibited by the CCK-A receptor antagonist L-364,718 and partially inhibited by the muscarinic cholinergic antagonist atropine. The ethanol-induced rise in amylase output can be completely prevented by instillation of trypsin into the duodenum or by lavage of the duodenum with saline during ethanol administration. Furthermore, the intraduodenal activity of a CCK-releasing factor is increased by infusion of ethanol. These studies indicate that administration of ethanol causes rat pancreatic exocrine secretion to increase. This phenomenon is mediated by a trypsin-sensitive CCK-releasing factor which is present within the duodenal lumen. These observations lead us to speculate that repeated CCK-mediated ethanol-induced stimulation of pancreatic digestive enzyme secretion may play a role in the events which link ethanol abuse to the development of pancreatic injury. (J. Clin. Invest. 1997. 99: 506-512.) Key words: pancreatitis • cholecystokinin • hormones $\bullet$ alcohol $\bullet$ cholecystokinin-releasing factor
\end{abstract}

\section{Introduction}

Ethanol abuse is an important factor in the etiology of pancreatitis but the mechanisms by which ethanol causes pancreatic injury have not been identified. Attempts to induce pancreatitis in experimental animals by either short-term or prolonged administration of ethanol have failed.

Address correspondence to Dr. Michael L. Steer, Department of Surgery, Beth Israel Hospital, 330 Brookline Avenue, Boston, MA 02215. Phone: 617-667-4261; FAX: 617-667-8679.

Received for publication 22 July 1996 and accepted in revised form 26 November 1996.

J. Clin. Invest.

(C) The American Society for Clinical Investigation, Inc.

0021-9738/97/02/0506/07 \$2.00

Volume 99, Number 3, February 1997, 506-512
A recent review of studies evaluating the effects of ethanol administration on pancreatic function has indicated that those effects depend on the species being studied and the route of ethanol administration. Thus, administration of ethanol has been associated with stimulation, inhibition, and no change in exocrine pancreatic secretion (1). For the most part, those previous studies have not defined the mechanisms responsible for the observed changes in exocrine pancreatic function in response to ethanol administration.

The present studies were undertaken in the belief that an understanding of the mechanisms by which ethanol administration alters pancreatic function might provide important clues to the mechanisms by which ethanol abuse causes pancreatitis. We have given rats ethanol in amounts sufficient to raise blood ethanol concentration to levels which have been clinically defined as intoxication. When given to either conscious or anesthetized rats under these conditions, we have found that ethanol causes cholecystokinin $(\mathrm{CCK})^{1}$-mediated stimulation of digestive enzyme secretion from the pancreas. Our observations indicate that administration of ethanol increases the activity of a trypsin-sensitive CCK-releasing factor in the duodenum and this, in turn, results in enhanced CCK release into the circulation.

\section{Methods}

All experiments were performed using male Wistar rats (200-300 g) purchased from Charles River Laboratories (Wilmington, MA). The animals were housed in temperature-controlled $23 \pm 2^{\circ} \mathrm{C}, 12$-h light/ dark rooms. They were given free access to rat chow and water. Caerulein was obtained from Research Plus, Inc. (Bayonne, NJ). The agents used to measure amylase activity and blood ethanol levels were obtained from Sigma Chemical Co. (St. Louis, MO). All other chemicals and reagents were of the highest purity available. The animals were maintained and all experiments were performed using protocols approved by the Institutional Animal Care and Use Committee of the Beth Israel Hospital.

\section{Animal preparation}

Blood alcohol levels. The protocol used to establish elevated but stable blood alcohol levels for subsequent experiments was evaluated using anesthetized rats. After an overnight fast, rats were anesthetized with an intraperitoneal injection of pentobarbital sodium (40 $\mathrm{mg} / \mathrm{kg}$ ) and anesthesia was maintained by periodic intravenous administration of pentobarbital sodium $(10 \mathrm{mg} / \mathrm{kg})$. Cannulae (PE50) were placed in each jugular vein. Ethanol was infused as shown in Fig. 1, through one of these cannulae and, at selected times, blood was withdrawn from the other cannula into a heparinized tube for blood alcohol measurement.

Pancreatic secretion in anesthetized rats. Rats were fasted overnight and anesthetized as described above. A cannula (PE50) was placed in the right jugular vein and used subsequently for ethanol in-

1. Abbreviation used in this paper: $\mathrm{CCK}$, cholecystokinin. 
fusion. A midline celiotomy was performed and, the common pancreatic-bile duct was cannulated extraduodenally with a PE10 catheter through which pancreatic secretions were collected. The bile duct was ligated on the liver side of the pancreas to prevent the flow of bile into the common pancreatic-bile duct. Core temperature was maintained using a heating pad and overhead lamp. The animals were allowed to stabilize for $30 \mathrm{~min}$ before the onset of ethanol infusion and collection of pancreatic juice. Ethanol was infused intravenously as shown in Fig. 1. Control animals received an identical volume of saline intravenously. In some animals $25 \%$ ethanol (or saline in controls) was injected directly into the stomach to achieve a blood alcohol level of $0.10 \mathrm{mg} \%$. This level of blood alcohol was maintained for the duration of the experiment by periodic intragastric administration of ethanol. Pancreatic secretions were collected over various time intervals in preweighed vials and volume output was calculated assuming a juice density of $1 \mathrm{~g} / \mathrm{ml}$.

Pancreatic secretion in conscious rats. $1 \mathrm{~d}$ before each experiment, fasted animals were anesthetized and cannulae were placed in the jugular vein as well as the common pancreatic-bile duct as described above. An additional cannula (PE50) was placed into the second portion of the duodenum and used for return of biliopancreatic secretions. The pylorus was ligated to prevent acid entry into the duodenum and gastric secretions were diverted from the obstructed stomach through a PE150 cannula placed through a stab wound in the gastric fundus. All cannulae were exteriorized and the celiotomy wound was closed. The animals were placed in a restraining cage, allowed to awaken from anesthesia, and given free access to water. During this time, the pancreatic-bile duct cannula was connected to the duodenal infusion cannula and, as a result, pancreatic as well as biliary secretions were not diverted from the duodenum. Each experiment was begun by determining basal (i.e., unstimulated) amylase output at 15-min intervals for $1 \mathrm{~h}$. At that time, animals were given either ethanol or saline intravenously and amylase output was measured at 15 -min intervals for $90 \mathrm{~min}$. For these measurements, the pancreatic-bile duct cannula was disconnected from the duodenal cannula for $30 \mathrm{~s}$ to permit sampling of the secretions and then the two cannulae were reconnected to avoid prolonged diversion of secretions from the duodenum. Since in these experiments the bile duct had not been ligated, the collected secretions consisted of bile as well as pancreatic juice and no significant change in volume was noted when basal and ethanol-stimulated conditions were compared. As a result, measurement of amylase concentration in the collected samples permitted calculation of changes in amylase output.

Bioassay of CCK-releasing factor. The effects of ethanol administration on duodenal CCK-releasing factor activity was evaluated using a "donor-recipient rat" bioassay system similar to that described by $\mathrm{Lu}$ et al. (2). "Donor rats" were prepared as described above for the secretion studies involving anesthetized rats. Additional cannulae were placed in the proximal duodenum (for infusion of lavage fluid) and $20 \mathrm{~cm}$ distally (for collection of lavage fluid). The rats were given intravenous saline (control) or ethanol as described in Fig. 1 for $1 \mathrm{~h}$ and, during that time, the duodenum was continuously lavaged in $\mathrm{pH}$ 7.4 phosphate buffer at a rate of $2.5 \mathrm{ml} / \mathrm{min}$. The collected lavage fluid was concentrated using an Amicon YM-3 filter and then dialyzed, overnight, at $4^{\circ} \mathrm{C}$ against phosphate-buffered saline (pH 7.4). "Recipient rats" were anesthetized but did not receive jugular vein catheters. A collection cannula was placed, extraduodenally, in the pancreatic-bile duct and the bile duct was ligated on the liver side of the pancreas. $1 \mathrm{ml}$ of saline containing soybean trypsin inhibitor $(10 \mathrm{mg} /$ $\mathrm{ml}$ ) was injected into the duodenal lumen and pancreatic secretions collected over the subsequent $5 \mathrm{~h}$. Under these conditions, amylase output increases transiently because of the absence of proteolytic enzyme activity in the duodenum but it then decreases to basal levels. When amylase output had returned to basal level, $1 \mathrm{ml}$ of the concentrated, dialyzed duodenal lavage fluid obtained from either saline(control) or ethanol-infused donor rats was instilled into the duodenum of the recipient animal, and the subsequent amylase output from the recipient rat over the next 120 min was determined.

\section{Bioassay of plasma CCK}

Experimental animals were killed by administration of a lethal dose of sodium pentobarbital. Blood $(6 \mathrm{ml})$ was collected in cold, heparinized tubes, by cardiac puncture. Plasma was prepared and CCK levels quantitated by bioassay as described by Liddle et al. (3) using freshly prepared pancreatic acini (4) obtained from uninstrumented but fasted rats. A standard curve for caerulein-stimulated amylase secretion from each acini preparation was established and the concentration of $\mathrm{CCK}$ in the plasma extracts was calculated using this standard curve. CCK levels were expressed, in picomolars, as caerulein equivalents. Recovery of known amounts of caerulein added to control rat plasma was evaluated with each experiment and found to vary from 80 to $105 \%$. The results of each experiment were corrected using the measured recovery rate for that experiment. Amylase releasing activity (i.e., CCK) measured in the plasma extracts was consistently and completely inhibited by addition of the CCK-A receptor antagonist L-364,718 to the bioassay.

\section{Other assays}

Blood ethanol was measured as described by Bucher and Redetzki (5). Amylase activity was quantitated as described by Pierre et al. (6) using maltotetrose as the substrate.

\section{Analysis of data}

The results reported in this communication represent the mean \pm SEM values obtained from four or more animals in each group. The significance of changes was evaluated using Student's $t$ test and significance defined as differences associated with $P<0.05$.

\section{Results}

Effects of ethanol administration on blood ethanol concentration. Infusion of ethanol, according to the protocol described

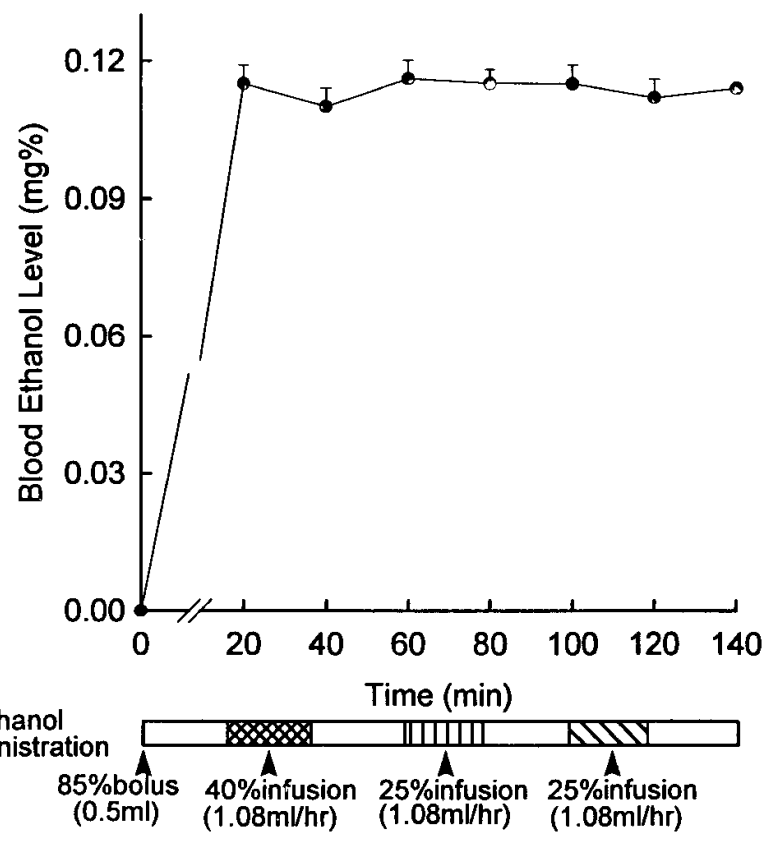

Figure 1. Blood ethanol levels during intravenous ethanol infusion. Rats were prepared as described in the text and, at time 0 , a bolus intravenous injection of $0.5 \mathrm{ml}$ of $85 \%$ ethanol was given. Between times 20 and $40 \mathrm{~min}, 40 \%$ ethanol was infused at a rate of $1.08 \mathrm{ml} / \mathrm{h}$. Between times 60 and $80 \mathrm{~min}$ and between times 100 and $120 \mathrm{~min}$, $25 \%$ ethanol was infused at a rate of $1.08 \mathrm{ml} / \mathrm{h}$. Blood ethanol was measured as described in the text. Results represent mean \pm SEM values from four to six animals evaluated at each time point 
A

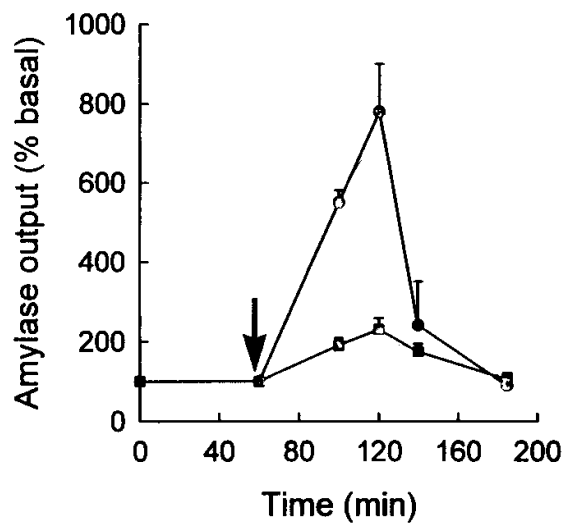

B

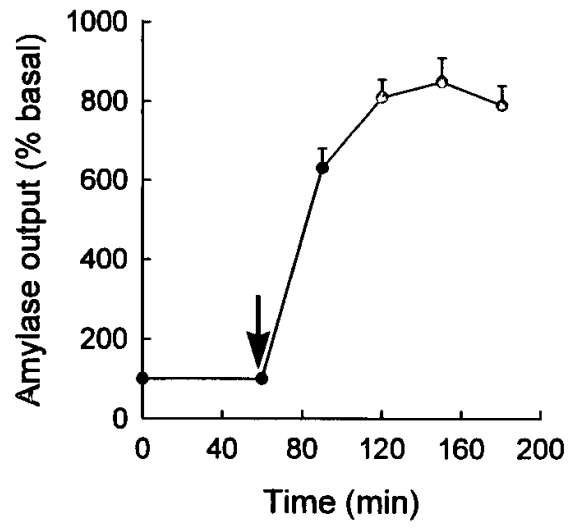

Figure 2. Effect of intravenous ethanol administration and caerulein on pancreatic output of amylase. $(A)$ Rats were given ethanol (circles) or saline (boxes) as described in Fig. 1 beginning at the time indicated by the arrow and pancreatic amylase output was measured as described in the text. $(B)$ In another group of animals prepared as in $A$ but not given ethanol, caerulein was infused beginning at the time indicated by the arrow and pancreatic amylase output was measured. Results represent mean \pm SEM for four or more animals evaluated at each time. in Fig. 1, in anesthetized rats resulted in a rise in blood ethanol concentration to a value of $0.12 \pm 0.03 \mathrm{mg} \%$. This rise was noted within $20 \mathrm{~min}$ of starting the infusion and the blood ethanol concentration remained at this level for the duration of the experiment (Fig. 1).

Effects of ethanol administration on pancreatic secretion in anesthetized rats. Infusion of ethanol resulted in a prompt stimulation of pancreatic amylase output (Fig. 2 A). Within 45 to $60 \mathrm{~min}$ of starting the ethanol infusion, amylase output reached a value which was seven- to ninefold greater than that noted before ethanol infusion and significantly greater than that observed for control animals infused with saline whose pancreatic juice had been similarly diverted from the duodenum by cannulation of the pancreatic duct. The small increase in amylase output observed in control animals is due to diversion of pancreatic secretions. As noted by others, diversion of pancreatic secretions from the duodenum results in decreased activity of trypsin in the duodenum and thus increases the activity of CCK-releasing factor $(2,7)$. Infusion of a volume of saline that was equivalent to that of the ethanol administered or infusion of the same volume of saline containing sufficient amounts of mannitol to raise the plasma osmolarity by 22 mosmol (the rise expected to occur if the blood ethanol concentration is $0.12 \%$ ) did not increase amylase output (data not shown). These findings indicate that the ethanol-induced increase in amylase output is not due to either an acute volume expansion or to an increased osmotic pressure.

In contrast to the blood ethanol level which remained constant during the period of ethanol infusion (Fig. 1), the amylase output was only transiently elevated during the ethanol infusion and, within 120 min of starting the ethanol infusion, amylase output had returned to the basal (i.e., preethanol) level (Fig. 2 A). Although ethanol infusion was noted to cause only a transient rise in amylase output, infusion of the CCK analogue caerulein in other animals was associated with a sustained rise in amylase output (Fig. 2 B). Furthermore, a substantial rise in amylase output induced by caerulein was also noted when caerulein was given to animals infused, as shown in Fig. $2 A$, with ethanol (not shown). Intragastric administration of ethanol also resulted in similar transient increase in amylase output (Fig. 3). These observations indicate that ethanol infusion causes only a transient rise in amylase output al-

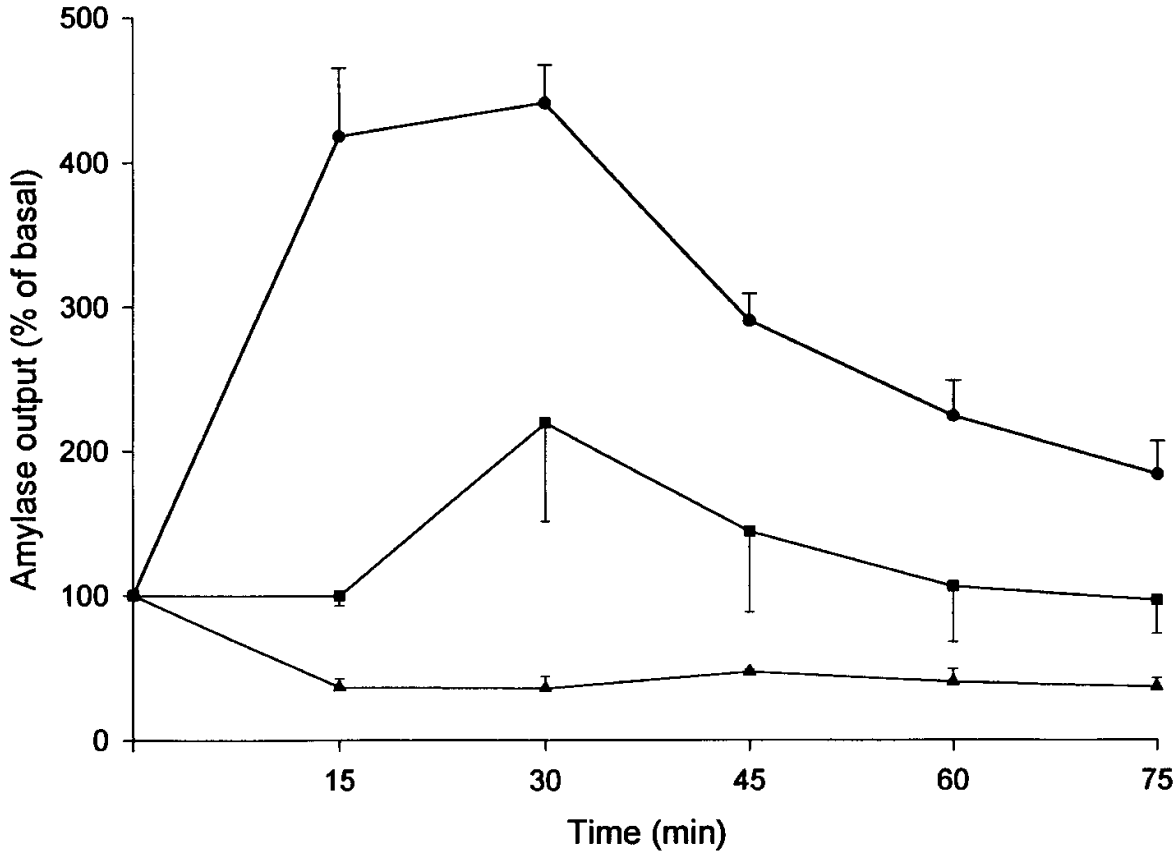

Figure 3. Effect of intragastric ethanol administration on pancreatic output of amylase. Rats were administered either intragastric saline (boxes) or ethanol alone (circles) or ethanol plus L-364,718 (triangles) and amylase output was measured as described in the text. Results indicate average values obtained from three or more animals at each time point. 
A

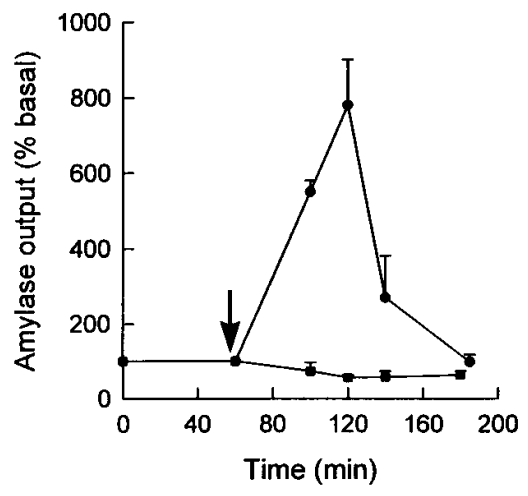

B

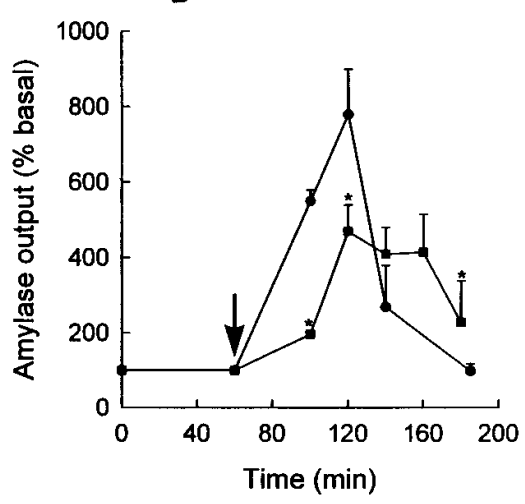

Figure 4. Effects of L-364,718 and of atropine on ethanol-stimulated output of amylase. Rats (boxes) were prepared as described in the text and given $5 \mathrm{mg} / \mathrm{kg}$ L-364,718 $(A)$ or $8 \mathrm{mg} / \mathrm{kg}$ atropine $(B)$. At the time indicated by the arrow, ethanol administration as described in Fig. 1 was begun. Control animals (circles) were treated identically but were given neither L-364,718 nor atropine. Amylase output was quantitated as described in the text. Results indicate values obtained from four or more animals at each time. Asterisks in $B$ indicate value for animals given atropine which is significantly different from that noted for animals not given atropine. though (a) ethanol levels remain constantly elevated; (b) the pancreas retains the capability of sustaining an increased rate of amylase output if suitably stimulated under these conditions; and (c) ethanol infusion by itself does not interfere with the ability of the pancreas to respond to stimulation of secretion.

Effects of L-364,718 and atropine on ethanol-stimulated amylase secretion in anesthetized rats. Amylase secretion stimulated by infusion of ethanol in anesthetized rats was completely inhibited by administration of the CCK-A receptor antagonist L-364,718 (Figs. 3 and 4 A). Administration of the cholinergic receptor antagonist atropine $(8 \mu \mathrm{g} / \mathrm{kg} / \mathrm{h})$ delayed the onset and reduced, by $\sim 50 \%$, the magnitude of the rise in amylase output induced by ethanol infusion (Fig. $4 \mathrm{~B}$ ). Higher concentrations of atropine $(100 \mu \mathrm{g} / \mathrm{kg} / \mathrm{h})$ had a similar but not a greater effect on amylase output (not shown).

Effect of ethanol administration on amylase secretion in conscious rats. For these experiments, pylorus-ligated animals were prepared and amylase secretion was evaluated under conditions which did not mandate diversion of biliopancreatic secretions from the duodenum. As shown in Fig. 5, infusion of ethanol in these conscious animals resulted in a transient 3.5- fold rise in pancreatic amylase output. No increase in amylase output was associated with intravenous saline infusion in control animals and the rise in amylase output associated with ethanol administration was completely prevented by the CCK-A receptor antagonist L-364,718.

Effects of ethanol infusion on plasma CCK levels in anesthetized rats. Ethanol infusion in anesthetized rats was associated with a transient rise in plasma CCK concentration (Fig. 6). Plasma CCK rose from a preethanol value of $0.8 \pm 0.4 \mathrm{pM}$ to a peak value of $39 \pm 10 \mathrm{pM}$ which was noted 45 min after starting the ethanol infusion. Thereafter, CCK levels rapidly declined and, although the blood ethanol concentration remained stably elevated (Fig. 1), the plasma CCK level had returned to the preethanol value of $1.0 \pm 0.5 \mathrm{pM} 120 \mathrm{~min}$ after starting the ethanol infusion. A modest but significantly lower elevation of plasma CCK levels was noted in the control animals which, like those infused with ethanol, had pancreatic secretions diverted from the duodenum as a result of pancreatic duct cannulation.

Effects of intravenous ethanol on CCK-releasing factor activity in the duodenum. As shown in Table I, the ethanolinduced rise in plasma CCK levels was completely inhibited

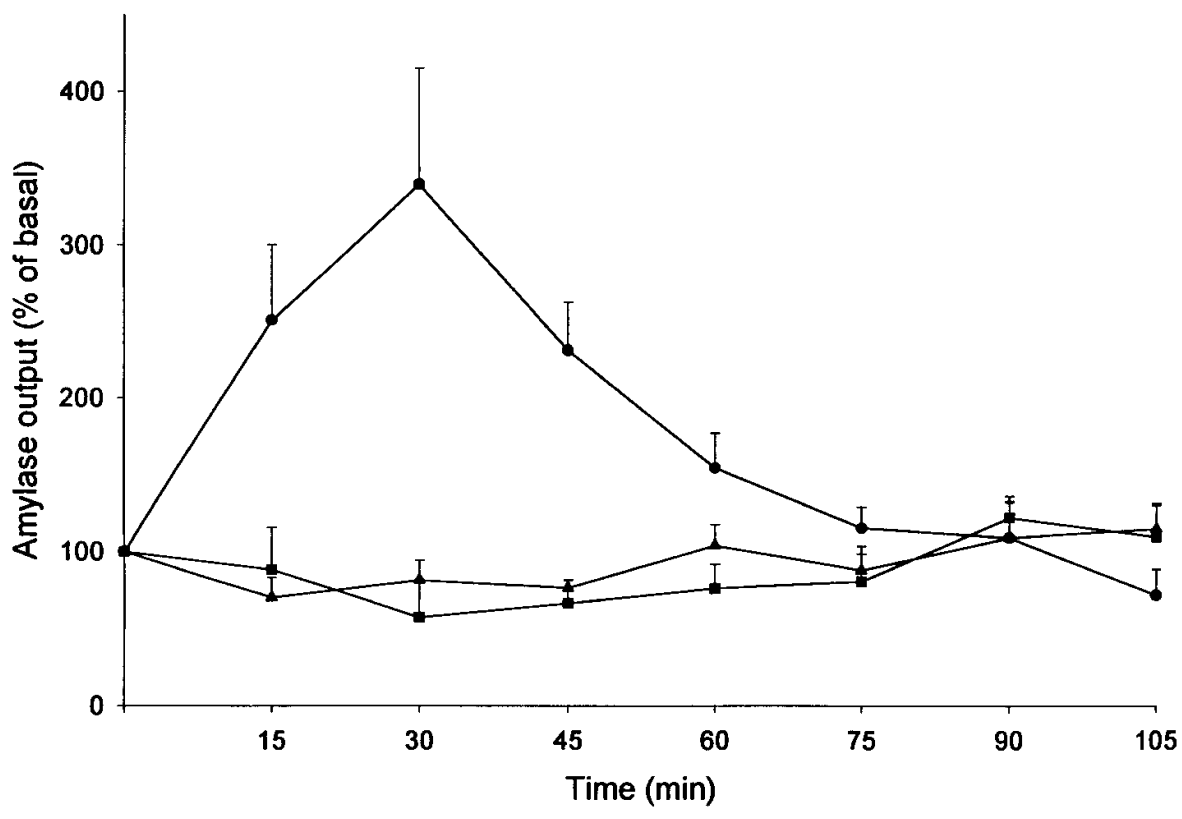

Figure 5. Effect of intravenous ethanol administration on amylase secretion in conscious rats. Pylorus-ligated conscious animals were infused with either saline (boxes) or ethanol alone (circles) or ethanol plus L-364,718 (triangles) and amylase output was measured as described in the text. Results indicate average values obtained from four or more animals at each time point. 


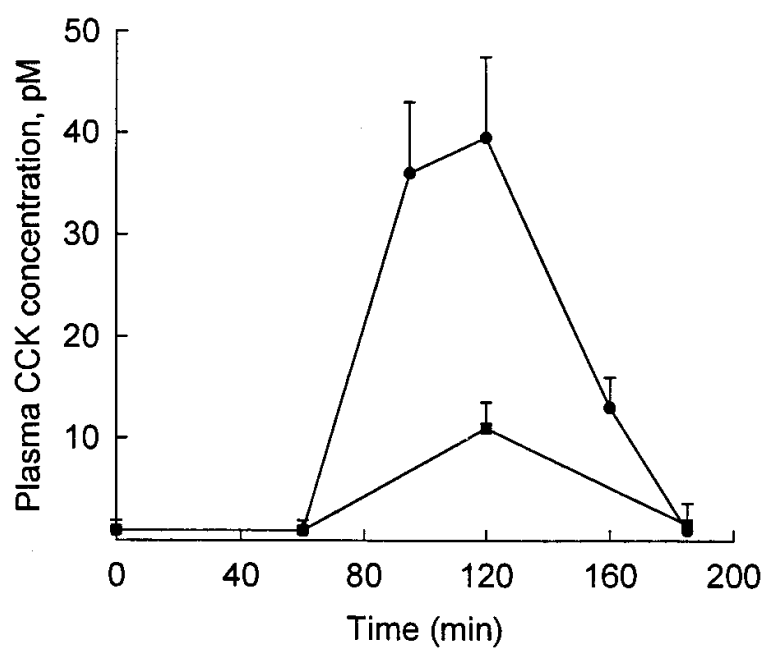

Figure 6. Effects of intravenous ethanol administration on plasma CCK levels. Rats were prepared and given ethanol (circles) or saline (boxes) as described in Fig. 1. The ethanol administration was begun at the time $=60 \mathrm{~min}$. Plasma CCK, expressed as picomolars but measured as caerulein equivalents, was quantitated by bioassay as described in the text. Results represent mean \pm SEM values for four or more animals evaluated at each time.

when trypsin was instilled into the duodenum. A similar inhibition was observed when the duodenum was lavaged with saline during the period of ethanol infusion. Under these conditions, both amylase output and plasma CCK levels were noted to be even lower than that noted in control animals not infused with ethanol.

Table I. Effect of Trypsin Infusion and Duodenal Lavage on Ethanol-stimulated Amylase Secretion and Rise in Plasma CCK

\begin{tabular}{lcc}
\hline \multicolumn{1}{c}{ Condition } & Amylase output & Plasma CCK \\
\hline & $\%$ of basal & $p M$ \\
Basal & 100 & $1.0 \pm 0.5$ \\
Saline & $230 \pm 45$ & $11.5 \pm 1.5$ \\
Ethanol & $750 \pm 136$ & $42.0 \pm 6.7$ \\
Ethanol + trypsin & $75 \pm 27^{*}$ & $2.0 \pm 1.0^{*}$ \\
Ethanol + lavage & $80 \pm 18^{*}$ & $3.5 \pm 1.2^{*}$
\end{tabular}

Rats were prepared as described in the legend to Fig. 2 with additional cannulae placed (a) in the gastric antrum to divert gastric secretion; (b) in the proximal duodenum for infusion of lavage fluid (phosphate buffered saline, $\mathrm{pH}$ 7.4); and (c) $20 \mathrm{~cm}$ distally in the jejunum to collect the lavage effluent. Amylase output and plasma CCK levels were measured during a 30-min basal (unstimulated) period before saline or ethanol infusion. Thereafter, animals were given an intravenous infusion of saline (control group) or ethanol with or without intraduodenal instillation of trypsin $(5 \mathrm{mg})$ or duodenal lavage with saline $(2.5 \mathrm{ml} / \mathrm{min})$ for an additional $1 \mathrm{~h}$. Amylase output and plasma CCK concentration were determined. Amylase output is expressed as a percentage of that found for the basal period. The modest rise in amylase output and plasma CCK concentration noted in the control group reflects the effects of diverting pancreatic secretions from the duodenal lumen. Four or more animals were used in each group and the results shown are mean \pm SEM for each measurement. $* P<0.05$ when the ethanol and trypsin or the ethanol and lavage groups were compared with the ethanol alone group.

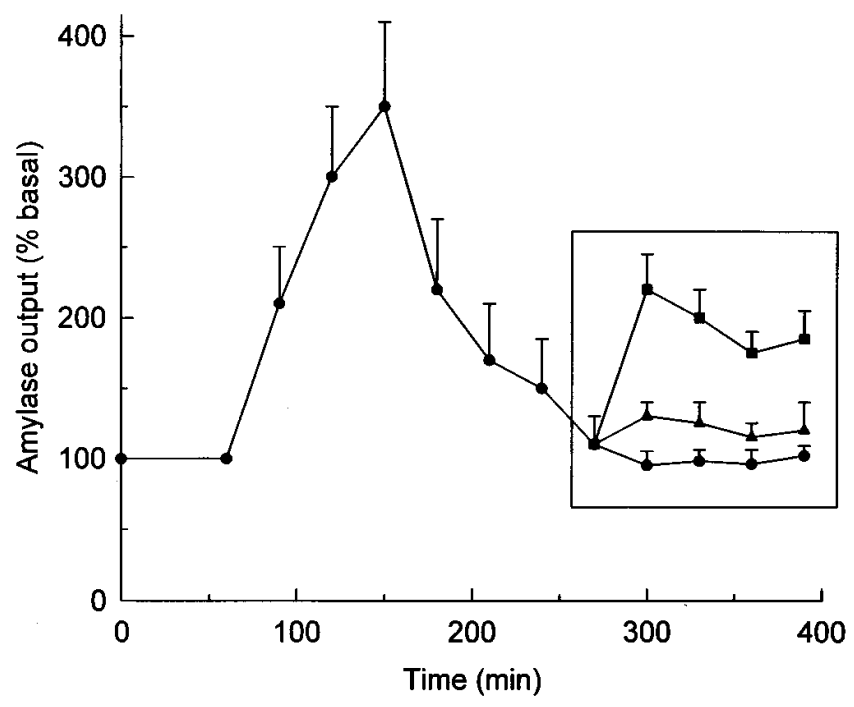

Figure 7. Effects of ethanol on duodenal CCK-releasing factor activity. For this experiment the recipient rats were prepared by diverting pancreatic secretions from the duodenum and the residual proteolytic activity in the duodenum was inhibited by soybean trypsin inhibitor. After amylase output returned to basal levels, concentrated duodenal lavage obtained from either saline (triangles) or ethanol (boxes) infused donor animals was instilled into the duodenum of some of the recipient rats (inset) and amylase output was quantitated as described in the text. Results shown are mean \pm SEM values obtained from four animals in each group.

The results of the donor-recipient animal experiments designed to directly evaluate duodenal CCK-releasing factor activity by bioassay are shown in Fig. 7. Recipient animals were prepared by diverting pancreatic secretions from the duodenum and inhibiting residual proteolytic activity in the duodenum with soybean trypsin inhibitor. This pretreatment resulted in a transient rise in pancreatic amylase output which gradually fell to basal levels within $5 \mathrm{~h}$. At that time, concentrated duodenal lavage fluid obtained from either saline or ethanol-infused donor rats was instilled into the duodenum of these recipient animals. A modest rise in amylase output was noted when lavage fluid from saline-injected donor rats was used but a significantly greater rise was observed when the lavage fluid from ethanol-infused donor rats was placed into the duodenum of the recipient animals (Fig. 7, inset).

\section{Discussion}

The protocol for ethanol administration used in our experiments was designed to result in blood ethanol levels which would equal or exceed those associated with clinical intoxication and which would remain stably elevated for the entire 2-h period of ethanol administration. The combination of bolus and intermittent infusions of varying concentrations of ethanol, by allowing for equilibration of ethanol among various fluid compartments as well as for ethanol metabolism, appears to have achieved this goal (Fig. 1). Using this protocol, blood ethanol levels rose to $0.12 \mathrm{mg} \%$ and remained at that level for the duration of each 2-h experiment.

The rise in blood ethanol concentration after intravenous or intragastric administration of ethanol to anesthetized rats 
was associated with a marked increase in the rate of amylase secretion from the pancreas. Surprisingly, the acceleration of amylase secretion induced by ethanol infusion was only transient although, under the conditions of our experiments, the blood ethanol concentration remained elevated and the pancreas remained responsive to a secretagogue such as the CCK analogue caerulein (Fig. 2). Within 120 min of starting the ethanol infusion, amylase output had returned to the preethanol level.

The stimulation of pancreatic amylase secretion associated with intravenous administration of ethanol to anesthetized rats was also noted when anesthetized rats were given ethanol intragastrically and when conscious rats were given intravenous ethanol (Fig. 5). Under the latter conditions, the magnitude of the response to ethanol administration was somewhat reduced, perhaps because pancreatic secretions had not been diverted from the duodenum in the conscious group. As had been noted with anesthetized rats, the ethanol-induced rise in amylase secretion from the pancreas of conscious animals was also transient and, by $75 \mathrm{~min}$ after the start of ethanol infusion, pancreatic amylase secretion had returned to the preethanol rate.

The transient pattern of amylase secretion associated with administration of ethanol to either anesthetized or conscious rats suggested to us the possibility that ethanol might accelerate the rate of enzyme secretion by an indirect mechanism rather than by a direct effect of ethanol on acinar cells. Previous studies by Liddle et al. (3) have indicated that intragastric instillation of ethanol in rats could cause plasma CCK levels to rise. We considered the possibility that intravenous administration of ethanol might also cause CCK levels to rise and that this might be the mechanism by which ethanol stimulates pancreatic amylase secretion. Indeed, the finding that the ethanolinduced rise in amylase secretion can be blocked in both anesthetized and conscious rats by the CCK-A receptor antagonist L-364,718 (Figs. 3, 4 A, and 5) is compatible with this conclusion. The finding that the cholinergic antagonist atropine can partially inhibit the ethanol-induced stimulation of amylase secretion (Fig. $3 \mathrm{~B}$ ) is also compatible with this conclusion since, as recently noted by others $(8,9)$, physiological concentrations of CCK accelerate pancreatic digestive enzyme secretion via an indirect, atropine-sensitive pathway which may involve intrapancreatic muscarinic cholinergic neurons. Further evidence that the ethanol-induced acceleration in amylase secretion from the pancreas reflects an ethanol-induced rise in plasma CCK levels comes from our studies which quantitated plasma CCK levels during intravenous ethanol infusion. As shown in Fig. 6, ethanol infusion results in a prompt and marked rise in plasma CCK levels which, like the ethanol-induced acceleration in amylase secretion, is transient. Within 120 min of starting the ethanol infusion, plasma CCK levels fall to the preethanol value.

Taken together, these various observations indicate that administration of ethanol to either anesthetized or conscious rats causes a transient rise in plasma CCK levels and, as a result, a comparably transient rise in pancreatic amylase secretion. CCK is synthesized and released into the circulation by specialized I cells within the mucosa of the duodenum and proximal jejunum (10). Release of CCK into the circulation from those cells is known to be regulated by a luminally active, trypsin-sensitive factor which has been called CCK-releasing peptide $(2,7)$. We have noted that the ethanol-induced rise in plasma CCK level as well as the ethanol-induced stimulation of pancreatic amylase secretion can be prevented by either placing trypsin into the duodenum or, alternatively, by lavaging the duodenum during the period of ethanol infusion (Table I). Both of these interventions would be expected to reduce the intraduodenal activity of the CCK-releasing factor and, therefore, our observations indicate that these effects of ethanol infusion are mediated by that CCK-releasing factor.

Two explanations for our observations should be considered. First, ethanol infusion might result in enhanced CCK release from I cells by increasing the sensitivity of those cells to a fixed level of CCK-releasing factor within the duodenum. The second explanation for our observations is the possibility that ethanol infusion might increase the intraduodenal level of the CCK-releasing factor. To discriminate between these two possibilities, bioassay of the CCK-releasing factor during ethanol infusion was carried out using the donor-recipient system. As noted in Fig. 7, CCK releasing activity is detectable in the duodenal lavage solution obtained from control, saline-infused, donor rats presumably because those animals have been exposed to conditions which diminish trypsin activity within the duodenum, i.e., diversion of pancreatic secretions and instillation of soybean trypsin inhibitor into the duodenum. In contrast to the small rise in CCK releasing activity noted in the control samples, the CCK releasing activity is markedly increased in the duodenal lavage solution obtained from donor rats that had received ethanol. These results clearly indicate that intravenous infusion of ethanol increases the intraduodenal level of CCK-releasing factor and strongly suggest that this is the mechanism by which ethanol administration results in a rise in plasma CCK levels as well as an enhanced rate of pancreatic amylase secretion.

Two distinct luminally active CCK-releasing factors have been described and extensively characterized $(2,7,11,12)$. One, referred to as monitor peptide, is secreted from acinar cells of the pancreas and is present in pancreatic juice while the other is released from as yet undetermined cells in the intestinal wall. Our finding that ethanol causes CCK-releasing factor activity to rise under conditions of juice diversion indicates that it is the latter rather than the former type of CCK-releasing factor which mediates the ethanol-induced rise in CCK levels and pancreatic enzyme secretion.

The results obtained in our studies may shed some light on the clinical situation in which toxic circulating levels of ethanol can be achieved. Since ingestion of ethanol increases intraduodenal CCK-releasing factor activity resulting in a transient rise in circulating CCK levels and stimulation of pancreatic acinar cell secretion, it would be intriguing to speculate that this intermittent and transient stimulation is, in some manner, deleterious to the pancreas. Long-term abuse of ethanol in humans has been noted to result in the secretion of pancreatic juice which is rich in protein, digestive enzymes, and lysosomal hydrolases $(13,14)$. Under appropriate conditions, trypsinogen can be activated by the lysosomal hydrolase cathep$\sin \mathrm{B}(15,16)$ and trypsin can activate the remaining zymogens. Thus, one might speculate that repeated but intermittent and transient CCK-mediated ethanol-induced stimulation of pancreatic secretion could lead to pancreatic injury by increasing the level of activated zymogens within the ductal space. Further studies, ideally using human subjects, should be designed to evaluate this possibility. 


\section{Acknowledgments}

The authors gratefully acknowledge the excellent technical support provided by N. Maitre.

This work was supported by National Institutes of Health grant DK-31396.

\section{References}

1. Singh, M., and H. Simsek. 1990. Ethanol and the pancreas: current status. Gastroenterology. 98:1051-1062.

2. Lu, L., D. Louie, and C. Owyang. 1989. Cholecystokinin releasing peptide mediates feedback regulation of pancreatic secretion. Am. J. Physiol. 257: 430-435.

3. Liddle, R.A., I.D. Goldfine, and J.A. Williams. 1994. Bioassay of plasma cholecystokinin in rats: effects of food, trypsin inhibitor and alcohol. Gastroenterology. 87:542-549.

4. Saluja, A.K., R. Dawra, M. Lerch, and M.L. Steer. 1992. CCK-JMV-180, an analogue of cholecystokinin, releases calcium from an inositol triphosphate independent pool in rat pancreatic acini. J. Biol. Chem. 167:11202-11208.

5. Bucher, T., and H. Redetzki. 1961. Eine spezlfische photometrische bestimmung von athylaikohol auf fermentatiuven wege. Klin. Wochenshr. 29:615

6. Pierre, K.J., K.N. Tung, and M. Nadi. 1976. A new enzymatic kinetic method for determination of amylase. Clin. Chem. 22:1219.

7. Miyasaka, K., D.F. Guan, R.A. Liddle, and G.M. Green. 1989. Feedback regulation by trypsin: evidence for intraluminal CCK-releasing peptide. Am. J. Physiol. 257:175-181.
8. Soudah, H.C., Y. Lu, W.L. Hasier, and C. Owyang. 1992. Cholecystokinin at physiological levels evokes pancreatic enzyme secretion via a cholinergic pathway. Am. J. Physiol. 263:G102-G107.

9. Adler, G., C. Berliner, U. Braun, M. Reinshagen, I. Koop, A. Schfmayer, L. Rovati, and R. Arnold. 1991. Interaction of the cholinergic system and cholecystokinin in regulation of endogenous and exogenous stimulation of pancreatic secretion in humans. Gastroenterology. 100:537-543.

10. Buchan, A., J. Polak, E. Solcia, C. Capelia, D. Hudson, and A. Pearse. 1978. Electron immunohistochemical evidence for the human intestinal I cell as the source of CCK. Gut. 19:403-407.

11. Spannagel, A.W., G.M. Green, D. Guan, R.A. Liddle, K. Faull, and J.R. Reeve. 1996. Purification and characterization of a luminal cholecystokininreleasing factor from rat intestinal secretion. Proc. Natl. Acad. Sci. USA. 93: 4415-4420.

12. Iwai, K., T. Fushiki, and S. Fokuoka. 1988. Pancreatic enzyme secretion mediated by novel peptide: monitor peptide hypothesis. Pancreas. 3:720-728.

13. Renner, I.G., H. Rinderknecht, and J.R. Wisner, Jr. 1983. Pancreatic secretion after secretin and cholecystokinin stimulation in chronic alcoholics with and without cirrhosis. Dig. Dis. Sci. 28:1889-1893.

14. Rinderknecht, H., I.G. Renner, and H.H. Koyama. 1979. Lysosomal enzymes in pure pancreatic juice from normal healthy volunteers and chronic alcoholics. Dig. Dis. Sci. 24:180-186.

15. Greenbaum, L.A., and A. Hirshkowitz. 1961. Endogenous cathepsin activates trypsinogen in extracts of dog pancreas. Proc. Soc. Exp. Biol. Med. 107: $74-76$.

16. Figarella, A., B. Miszczuk-Jamska, and A.J. Barrett. 1988. Possible lysosomal activation of pancreatic zymogens. Activation of both human trypsinogens by cathepsin B and spontaneous acid activation of human trypsinogen. $J$. Biol. Chem. Hoppe-Seyler. 369(Suppl.):293-298. 